\title{
Modelling: Compartmental Networks and Topologies A Comparison with Bond Graphs
}

\author{
Heinz A Preisig* \\ Department of Chemical Engineering; NTNU; Trondheim, Norway
}

\begin{abstract}
Compartmental network modelling is compared with the bond graph approach. Whilst both approaches build on the conservation principle, the compartmental network approach is on a slightly higher level and more compact. Also no analogies must be used, the concepts of physics are used directly in connection whilst system theoretical arguments define consistency and solvability, being roughly equivalent to causality in bond graphs.
\end{abstract}

Keywords: modelling, mechanistic, first principle, Bond Graphs

\section{Scope}

The author looks back to somewhere around 20 years of research in computer-aided modelling, which resulted in a methodological approach to modelling of physical-chemicalbiological systems implemented now in the third generation as a tool we call Modeller (Westerweele (2003)). The method builds on a compartmental network modelling approach and in the course of the last few years the question of the relation of this network approach with the bond graph approach has been raised a couple of times. Thus this paper is trying to illuminate some of the main differences. The views being reflected are personal and may not be shared by the bond graph specialist as both approaches are rather much building on macroscopic physics, meaning that the quantisation of matter and energy is not of relevance, but that it deals in general with a larger time scale and field theory applies in its full scope. It should be noted that modelling is here limited to defining a set of differential algebraic equations describing the behaviour of natural systems ${ }^{1}$.

\section{Compartmental Network Modelling}

The approach is based on splitting the relevant piece of the world into a set of communicating control volume. Thus we define a basic model topology as a mapping of the real world object into a network of primitive systems that can store EXTENSIVE CAPACITIES and Connections, which can transfer extensive quantities. This topology we call the Physical TOPOLOGY, as it represents the physical containment of the process. This first step cannot be automated.

\footnotetext{
*e-mail: Heinz.Preisig@chemeng.ntnu.no

${ }^{1}$ The limitation to natural is not necessary, but the extension is also not of relevance to this discussion.
} 
Each of the two elements in the physical topology is given a behaviour. The capacities are dynamic and are characterised by the change in the conserved extensive quantities ${ }^{2}$ such as component mass, energy and momentum, to mention the main ones. These extensive quantise are being labelled with the attribute fundamental. $\varphi$ is a generic member of a set of extensive quantities $\mathcal{E}$; thus $\varphi \in \mathcal{E}$ and the set of fundamental extensive quantities $\mathcal{E}^{f} \subset \mathcal{E}$ and its generic member $\Phi$ with instance $\Phi^{A}$ being the fundamental quantity of type A, thus $\Phi \in \mathcal{E}^{f} \subset \mathcal{E}$. The connections represent the flows exchanging (fundamental) extensive quantities between capacities. We use a hat ${ }^{\wedge}$ to distinguish flows from time derivatives, which are shown with the raised-dot notation ' used for the accumulation term. Typical flows are mass, heat, work. We also introduce the concept of induced flows, meaning that for example mass carries also internal, kinetic and potential energy as well as doing volume work.

Depending on the extensive quantity being relevant in the various parts, the physical topology can be coloured, which, in simplistic terms, indicates what extensive quantities are being seen as relevant and thus are included in the model and where in the model this is the case. The colouring process indicates wherever the particular quantity is present due to the definitions being made in the course of the model construction. In physical-chemicalbiological processes one of these colouring is associated with the species present in the system, which we refer to as the SPECIES TOPOLOGY. Equally well, one can talk about an energy colouring, or more restrictive heat-colouring.
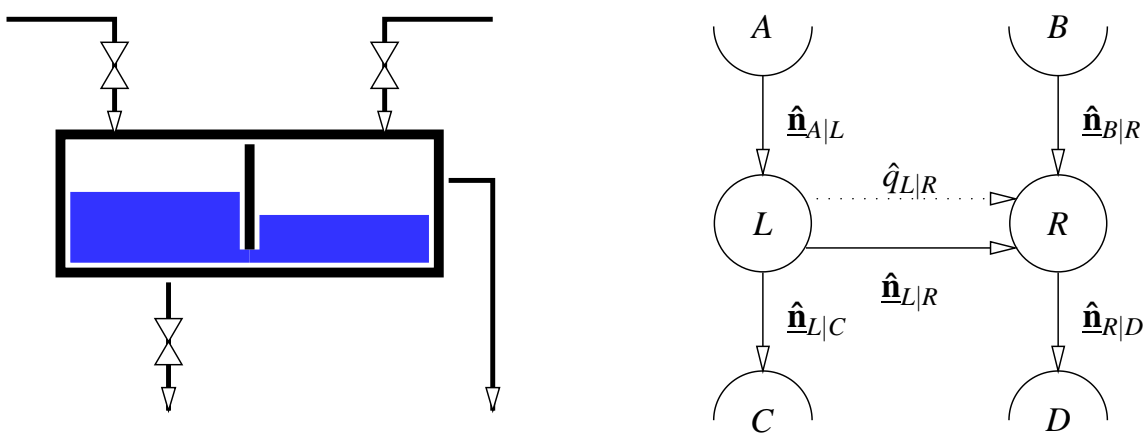

Figure 1. RHS: A sample plant; LHS: Physical topology, assuming (i) two lumped systems one for each compartments (ii) four reservoirs two as sources, two as sinks (iii) unidirectional flow for the flows in and out of the container, bidirectional mass flow inside, (iv) conductive heat flow through the wall, (v) no significant heat conduction through fluid flowing through opening, (vi) no heat or material losses.

In order to complete the description, each capacity and connection and the defined extensive quantities are associated with equations, which forms the EQUATION TOPOLOGY. The Physical Topology forms a directed graph with the nodes (circles) representing the capacities and the edge (arrow) representing the transfer of extensive quantity Figure 2 shows on the right the sample plant and on the left a possible physical topology. The graph is directed, that is, the edges have direction, which serve as reference co-ordinates

\footnotetext{
${ }^{2}$ Extensive quantise depend on the (physical) dimension of the system
} 
against which the dynamic flow is measured (+ in the same direction, - in opposite direction). Placing species A into reservoir A and species B into reservoir B, further assuming uni-directional flow between the reservoirs and the container as well as for the outflows, the species A will also present in compartment L \& $\mathrm{R}$ as well as in the two sink reservoirs. For species B it is the same except that it is modelled as not being present in reservoir A but rather in $\mathrm{B}$. The relevant fundamental extensive quantities are then the component masses and the total energy. We denote the component mass in system $i$, measured in moles, in a vector $\underline{\mathbf{n}}_{i}$ and the energy in the same system as $E_{i}$. The flow of mass from system $a$ to $b$ is denoted by $\underline{\hat{\mathbf{n}}}_{a \mid b}$.

$\frac{d \underline{\mathbf{n}}_{i}}{d t} \quad:=\underline{\underline{\mathbf{F}}}_{i}^{m} \underline{\hat{\mathbf{n}}}^{+} \underline{\underline{\mathbf{R}}}_{i}^{m} \underline{\tilde{\mathbf{n}}}_{i} \quad i:=L, R$.

The two matrices $\underline{\underline{\mathbf{F}}}_{i}^{m}$ and $\underline{\underline{\mathbf{R}}}_{i}^{m}$ are the flow and the transposition matrix ${ }^{3}$, both being typed, here with mass. The vector $\underline{\hat{\mathbf{n}}}$ is a stack of all component mass flow vectors in the system, namely $\underline{\hat{\mathbf{n}}}:=\left[\underline{\hat{\mathbf{n}}}_{A \mid L} ; \underline{\hat{\mathbf{n}}}_{B \mid R} ; \underline{\hat{\mathbf{n}}}_{L \mid R} ; \underline{\hat{\mathbf{n}}}_{L \mid C} ; \underline{\hat{\mathbf{n}}}_{R \mid D}\right]$. The two equations can also be stacked to form a description of the overall system:

$\frac{d \underline{\mathbf{n}}}{d t}:=\underline{\underline{\mathbf{F}}}^{m} \underline{\hat{\mathbf{n}}}+\underline{\underline{\mathbf{R}}}^{m} \underline{\tilde{\mathbf{n}}}$.

Energy balances are next: We usually distinguishe between three basic types of flows, namely energy associated with mass, that is internal, kinetic and potential energy, all captured in the "total energy", which is denoted by the symbol $E$. The second type is heat and the third work.

$\frac{d E_{i}}{d t} \quad:=\underline{\underline{\mathbf{F}}}_{i}^{m} \underline{\hat{\mathbf{E}}}+\underline{\underline{\mathbf{F}}}_{i}^{q} \underline{\hat{\mathbf{q}}}+\underline{\underline{\mathbf{F}}}_{i}^{w} \hat{\hat{\mathbf{w}}} \quad i:=L, R$.

It is customary and convenient to split the work term into subclasses. The split is not always done the same way, but most commonly one finds three parts convenient: (i) volume work associated with mass flowing across the boundary, (ii) volumetric work done by the system itself as it changes the volume, (iii) one the rest such as mechanical work etc. ${ }^{4}$ Using this classification the issue of induced flows becomes apparent, as mass flow induces not only a flow of internal energy, kinetic energy and potential energy, but also volumetric work:

$\frac{d E_{i}}{d t}:=\underline{\underline{\mathbf{F}}}_{i}^{m}\left(\underline{\hat{\mathbf{E}}}+\underline{\hat{\mathbf{w}}}^{m}\right)+\underline{\mathbf{F}}_{i}^{q} \underline{\hat{\mathbf{q}}}+\underline{\underline{\mathbf{F}}}_{i}^{r} \underline{\hat{\mathbf{w}}}^{r}+\underline{\hat{\mathbf{w}}}_{i}^{V} \quad i:=L, R$.

The split is done by typing the quantities. Here we used $m$ for mass, $r$ for the "rest", $V$ for the system's volume work. Without looking at any more details, one can now see clearly what role the typed subnetworks of the physical topology play in the formulation of the

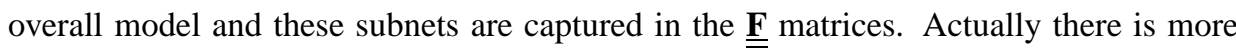
in it, as the typing extends down to the level of the individual extensive quantities being introduced, thus also the species distribution, that is the species topology, is captured in these matrices. This concept can be readily extended to any kind of extensive quantity.

\footnotetext{
${ }^{3}$ describes the ratio of converting one (fundamental) extensive quantity into others, used to describe reactions but also phase change

${ }^{4}$ The remaining work term may be further subdivided for example into surface tension may be of interest or anything other work term.
} 


\section{Bond Graphs and Its Derivatives}

Before we begin a word to the literature on bond graphs. Since this technology is around for nearly half a century, the literature body is rather large. However, there exist a couple of excellent textbooks, surveys and bibliographies that make the search for literature quite straightforward. On biographies the one of Breedveld and Rosenberg summarize the development up to 1991 nicely (Breedveld et al. (1991)). Another extensive source is currently available at the web site of Francoise Cellier (http://www.ece.arizona.edu/ cellier/).

\subsection{Basics}

Bond graphs derive historically from the need of mapping process models into analogue computing devices. In traditional bond graphs is thus the analogy of electrical networks and process models of all kinds in the centre (Breedveld (2003)). The modelling person has to translate the physical quantities being used in his process into analogue quantities that, initially being electrical quantities such as capacity, induction, resistance, electrical potential and current, later were abstracted to improve communications with others than the electrical engineers. The basics are, as in the network modelling approach, the CONSERVATION LAWS and the POSITIVENESS OF ENTROPY. For storage bond graphs introduce CAPACITORS and INDUCTORS; RESISTORS dissipate energy and SOURCES provide EFFORT and FLOW. Besides bonds, which represent power and have two components, namely EFFORT and FLOW, two types of ideal junctions are introduced: 0-JUNCTION and the 1 -JUNCTION. The 0 -junction passes the effort through, representing the equivalence of the connected effort variable, whilst the flows sum to zero with the signature being defined by the directionality of the connected bonds. It represents essentially Kirkhoff's current law. The 1-junction works the other way, the sum of the effort variables is zero again with the bond directionality defining the signature, whilst the flow is equal. This represents essentially Kirkhoff's voltage law (reference: any textbook on bond graphs).

\subsection{Analogies}

The key to the use of bond graphs is to think in analogies (which is also seen as a burden when changing its image extending to the bond graph concept (Breedveld, personal communications). The translation into mechanical \& electrical quantities and thus their use in such models, is relatively straightforward, though needs some exercising and getting used to. This explains the most common use of bond graphs in megatronic systems (Hogan and Breedveld (2002)). Field theory gives an easy and generic entry point to the analogy: temperature drives entropy flow, pressure drives mass flow, and voltage current, etc. However it yields an entry point only as a rather intriguing discussion by Hogan and Breedveld shows (Hogan and Breedveld (2002)). Leaving this reading and the associated thoughts to the interested reader, the field-theory point of view will be used in the remainder, as it does provide important insight. The effort variable is then to be interpreted as the potential difference, the driving force, whereby the potential is in thermodynamic sense the partial derivative of the fundamental function with respect to the fundamental extensive quantity that is flowing. For example, for entropy flow the potential is the temperature, which is the partial derivative of the internal energy with respect to entropy (Modell and 
Reid (1974)). With the electrical circuits being one-dimensional, the obvious problem arises when finding analogies for multi-dimensional systems. In mechanical systems it is the special co-ordinates and in chemical systems the number-of-species-dimensional species space that poses the problems. It is thus that for example Breedveld has been discussing thermodynamics as an alternative basis for the analogies (Breedveld (1984)), (Hogan and Breedveld (2002)).

\section{Consistency, Solvability, Causality \& Completeness}

Bond graph analysis uses the concept of CAUSALITY to insure solvability and thus also completeness of the representation within the limits of the assumptions made when establishing the model. This concept is very powerful and is further reaching than the common analysis made in, for example flow sheeting packages. It is an analysis that goes beyond the normal degree of freedom analysis and the DAE-index analysis.

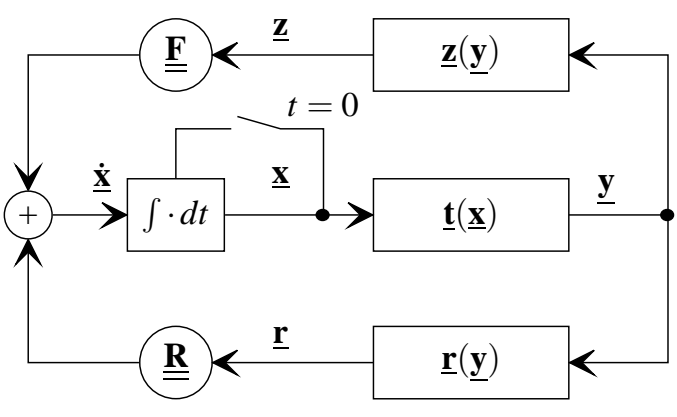

The $\underline{\mathbf{x}}$ is the state being the fundamental extensive quantities. Thus linearity and applies, which eventuates in the two matrices $\underline{\mathbf{F}}, \underline{\mathbf{R}}$ for the flow $\underline{\mathbf{Z}}$ and reaction feedback $\underline{\mathbf{r}}$ summing up to the accumulation term, the state's time derivative $\underline{\dot{\mathbf{x}}}$. Flows and reactions are a function of the secondary state $\underline{\mathbf{y}}$.

Figure 2. Block diagram of the overall equations system.

The Modeller project, as described in Westerweele $(2003)^{5}$, uses a system theoretical approach and distinguishes between completeness and solvability. Completeness is analysed based on the block diagram Figure 4. In short the analysis starts with the fundamental state $\underline{\mathbf{x}}$. The secondary state $\underline{\mathbf{y}}$ must be the result of a mapping from the $\underline{\mathbf{x}}$. Most commonly, the state variable transformations $\underline{\mathbf{x}}$ are not as simple as shown in the diagram but are also function of the secondary state. Usually these transformations are though (block) lower triagonal (Westerweele (2003)). The matrix $\underline{\underline{\mathbf{F}}}$ is the extracted from the network graph and the reaction matrix, or more general the transposition matrix $\underline{\underline{\mathbf{R}}}$ is defined through the reactions and other transposition mechanisms for fundamental extensive quantities. Requesting for every transfer and every reaction a proper expression completes the analysis. The analysis mechanics use bi-partite graph methods for multi-dimensional equations systems, which is an extension of the standard method.

Similar to the current bond graph theory, the initial conditions and parameters or known flows or reactions etc. must be specified before the analysis is made in the current implementation of the Modeller (Westerweele (2003)). The bond graph's causality argument

${ }^{5}$ more references may be found on the authors webpage 
works almost identical, though it looks rather different indeed. One has to look very carefully to identify the near identity of the two approaches.

\section{Conclusions}

From the reading and studying bond graphs, it appears that the network modelling approach worked out in out group over the last years is more general and addresses a number of issues still in question in bond graphs and pseudo bond graphs. Primarily, the network modelling approach does not depend on analogies, rather it implements the conservation principles in its most basic forms. It has an explicit handling of induced flows and allows for an on-line analysis of the model for consistency, thus consistency is always enforced, which is similar to the bond graph's causality analysis. The difference is mainly in the on-line versus batch analysis. Further, the limitation to analysing only simulation problems can be softened and removed in the future. The implemented network modelling approach is currently limited to component mass and energy. It could though readily be expanded to any type of conserved extensive quantity.

The main disadvantage of the bond graph world is its dependency on analogies. The rather cryptic graphical representation is gradually being replaced by a more friendly way of showing things. The fact that the port concept generates a rather large number of unnecessary cut equations is merely a technical detail, though the port concept is a level lower than the connection concept as it is used in the network modelling approach. Whilst the original bond graphs were really only for insiders, the development has been opening new avenues and the latest ideas as they are discussed for example in Hogan and Breedveld (2002) indicate that a more general view is slowly making bond graphs to migrate closer to the network modelling. Though as long as the analogies are an integral part, statements such as PERHAPS MOST IMPORTANT, IT LEADS TO ANALOGIS (ESPECIALLY BETWEEN MECHANICAL AND FLUID SYSTEMS) THAT DEFY COMMON PHYSICAL INSIGHT. (Hogan and Breedveld (2002)) will be a serious problem.

\section{References}

Breedveld, P. C.: 1984, Physical Systems Theory in Terms of Bond Graphs, PhD thesis, University of Twente, Enschede, Netherlands.

Breedveld, P. C.: 2003, Port-base modeling of mechatronic systems, MATHMOD 4, Vienna,Austria.

Breedveld, P. C., Rosenberg, R. C. and Zhou, T.: 1991, Bibliography of bond graph theory and application, Journal of the Franklin Institute 328(5/6), 1067-1109.

Hogan, N. and Breedveld, P. C.: 2002, The physical basis of analgies in physical system models, The Physical Basis of Analgies in Physical System Models, CRC Press, Boca Raton, London, New York, Washington DC.

Modell, M. and Reid, R.: 1974, Thermodynamics and its Applications, Prentice Hall, Englewood Cliffs, NJ.

Westerweele, M. R.: 2003, Five Steps for Building Consistent Dynamic Process Models and Their Implementation in the Computer Tool MODELLER, $\mathrm{PhD}$ thesis, TU Eindhoven, Eindhoven, The Netherlands. 